\title{
Second generation optical-resolution photoacoustic microscopy
}

Konstantin Maslov, Song Hu, Lihong V. Wang

Konstantin Maslov, Song Hu, Lihong V. Wang, "Second generation opticalresolution photoacoustic microscopy," Proc. SPIE 7899, Photons Plus Ultrasound: Imaging and Sensing 2011, 789933 (22 February 2011); doi: 10.1117/12.874786

SPIE. Event: SPIE BiOS, 2011, San Francisco, California, United States 


\title{
Second-generation optical-resolution photoacoustic microscopy
}

\author{
Konstantin Maslov ${ }^{\dagger}$, Song $\mathrm{Hu}^{\dagger}$, and Lihong V. Wang* \\ Optical Imaging Laboratory, Department of Biomedical Engineering, Washington \\ University in St. Louis, St. Louis, Missouri 63130, USA \\ ${ }^{\dagger}$ Authors contributed equally to this work. \\ *Corresponding author: $\underline{\text { lhwang@biomed.wustl.edu }}$
}

\begin{abstract}
We developed a second-generation optical-resolution photoacoustic microscope (OR-PAM) with a novel acoustic detection scheme, which improved upon the sensitivity of our first-generation system by $18 \mathrm{~dB}$. Moreover, translating the imaging head instead of the living object improved the scanning speed by a factor of 5 , widening the field of view within the same acquisition time. The anatomy and hemoglobin oxygen saturation of an entire mouse ear was imaged in vivo.
\end{abstract}

Keywords: Photoacoustic imaging, photoacoustic microscopy, high sensitivity, wide field of view.

\section{INTRODUCTION}

In many potential clinical applications it is necessary to image anatomic structures with high resolution, to identify structures according to their optical spectral properties, or to detect very small biomarkers such as conjugated nanoparticles. There is also a significant amount of research performed in vivo on animal models, such as studies of angiogenesis or cancer metastatic activity by monitoring of circulated cancer cells. In such research a minimally invasive imaging technique suitable for longitudinal studies with at least the cellular resolution is required.

As an emerging high-resolution imaging modality, photoacoustic microscopy (PAM) images optically absorbing microstructures by detecting transient acoustic waves generated from laser-induced thermal-elastic expansion. PAM is directly sensitive to optical absorption, which provides quantitative measurements of physiologically important endogenous molecules (e.g., hemoglobin and melanin) as well as a variety of widely used optical contrast agents (e.g., methylene blue and indocyanine green). In acoustic-resolution PAM systems, both the transverse and axial resolutions are defined by ultrasonic detection. However, because of the overwhelming attenuation of high-frequency ultrasound beyond $100 \mathrm{MHz}$ in biological tissue, it is difficult to achieve a transverse resolution below $10 \mu \mathrm{m}$ with ultrasonic focusing without severely compromising the imaging depth. To overcome this obstacle, we recently proposed opticalresolution PAM (OR-PAM), whose transverse resolution is defined by a tight optical focus. Using an optical microscope objective with an NA of 0.1 we experimentally obtained $5-\mu \mathrm{m}$ transverse resolution, and in vivo microvasculature was imaged down to the single-capillary level [1-6]. However, in our first-generation (G1) OR-PAM, the optical-acoustic combiner suffers significant acoustic loss [1]. Moreover, the G1 system could cover only a small field of view (FOV) within a reasonable acquisition time because translating the living object for scanning must be slow enough to avoid possible disturbance to the object. Here, we introduce our second-generation (G2) OR-PAM with improved detection sensitivity and imaging speed. In G2 OR-PAM, we have designed a novel optical-acoustic combiner, which improves the detection sensitivity by $18 \mathrm{~dB}$ over the G1 system. Moreover, the fiber-based light delivery allows scanning the imaging head rather than the living object, thereby enabling fast in vivo imaging over a large FOV.

\section{METHODS AND MATERIALS}

Our G2 OR-PAM [7], consists of both optical and ultrasonic components. The optical irradiation source is a dye laser pumped by a Nd:YLF solid-state pulsed laser. The output laser beam is attenuated by a neutral-density filter and coupled

Photons Plus Ultrasound: Imaging and Sensing 2011, edited by Alexander A. Oraevsky, Lihong V. Wang, Proc. of SPIE Vol. 7899, 789933 · C 2011 SPIE · CCC code: 1605-7422/11/\$18 · doi: 10.1117/12.874786 
into a single-mode optical fiber to allow mechanically scanning the microscope imaging head rather than the living object. To create a tight optical focus, the optical field of the single-mode fiber output is mapped into the imaging object by two identical microscope objectives. A beam sampler and a photodiode are inserted between the collimation objective and a stationary mirror to monitor the fluctuation in laser intensity. A CCD camera is added to view the imaging region through the reverse path of the optical illumination. The ultrasonic detection involves a home-made acoustic-optical beam combiner connected to $50-\mathrm{MHz}$ central frequency ultrasonic transducer. The combiner aligns the optical irradiation and the acoustic detection coaxially. Different from the G1 OR-PAM system, where two right-angle prisms sandwich a thin layer of silicone oil for acoustic-optical coaxial alignment [1], G2 OR-PAM replaces the lower rightangle prism with a rhomboid prism to enhance the detection sensitivity. In the G1 system, the obliquely incident $\left(45^{\circ}\right)$ acoustic signal is reflected by a single solid-liquid interface (Fig. 1), where the energy of the longitudinal acoustic wave is coupled into both shear and longitudinal waves. According to the boundary conditions, $85 \%$ of the incident acoustic energy is transformed from longitudinal mode to shear mode at the interface. The second inclined surface provided by the rhomboid prism in the G2 system is able to transform $97 \%$ of the shear-wave energy back into longitudinal waves, to which the ultrasonic transducer is sensitive. In comparison to the combiner in the G1 system, the new design can theoretically result in a $20-\mathrm{dB}$ increase in the detected longitudinal-wave energy. Our experimental results show that, at the same optical irradiation level, the signal-to-noise ratio (SNR) of the G2 system was $18 \mathrm{~dB}$ higher than that of the G1 system. The actual improvement in SNR is in good agreement with the theoretical estimation.

Into the bottom of the combiner a spherical cavity is ground to produce an acoustic lens with a numerical aperture of 0.5 in water, which provides an acoustic focal diameter of $43 \mu \mathrm{m}$ at the $50-\mathrm{MHz}$ central frequency. To maximize the detection sensitivity, the optical and acoustic foci are aligned confocally. The OR-PAM system also includes electronic amplifiers, an analog-digital converter, a mechanical scanner, and a scanner controller. The digitized signal is transferred to a computer for image formation. The software converts the raw data into a sequence of cross-sectional B-scan images as the microscope head raster scans the object. The recorded images can then be displayed as individual cross-sectional images, 2D maximum amplitude projection images, or 3D renderings. A water tank is used to house the microscope imaging head. We cut an imaging window into a Petri dish and seal it with an ultrasonically and optically transparent polyethylene membrane. The photoacoustic wave generated from the object is acoustically coupled to the ultrasonic detector, first by ultrasonic gel between the object and the polyethylene membrane and then by deionized water in the Petri dish.

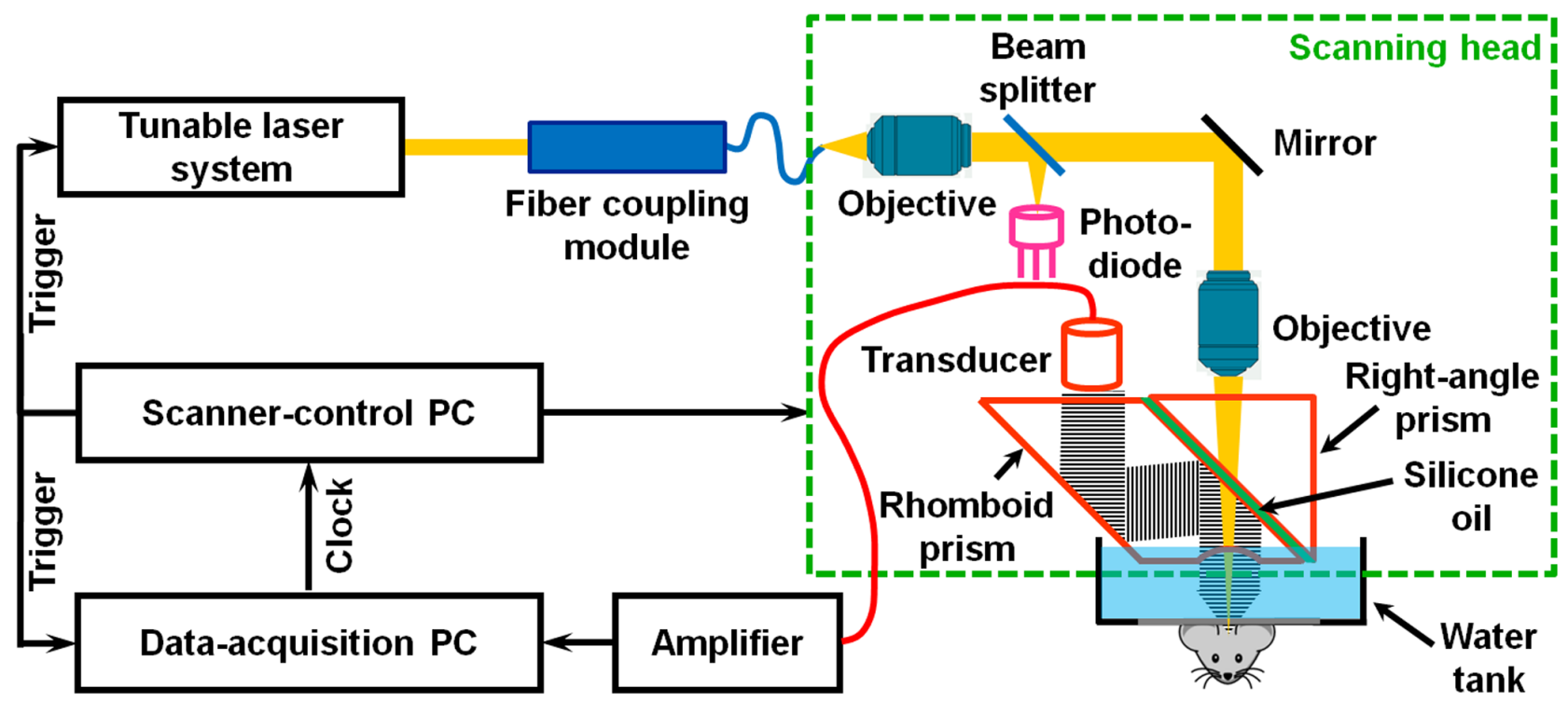

Figure 1. Experimental setup of the second-generation optical-resolution photoacoustic microscope.

The much improved imaging speed enables G2 OR-PAM to image a large FOV with single capillary resolution and sensitivity. Figure 2 shows the distributions of total concentration (Fig. 2A) and oxygen saturation (Fig. 2B) of 
hemoglobin in a living nude mouse ear. Densely packed capillary beds are clearly resolved (Fig. 2C). The typical acquisition time of such a dual-wavelength, bidirectionally scanned image (image size: $10 \mathrm{~mm} \times 10 \mathrm{~mm}$; step size: 2.5 $\mu \mathrm{m} \times 5 \mu \mathrm{m})$ is $\sim 80 \mathrm{~min}$, which is 5 times as fast as that for the G1 system.
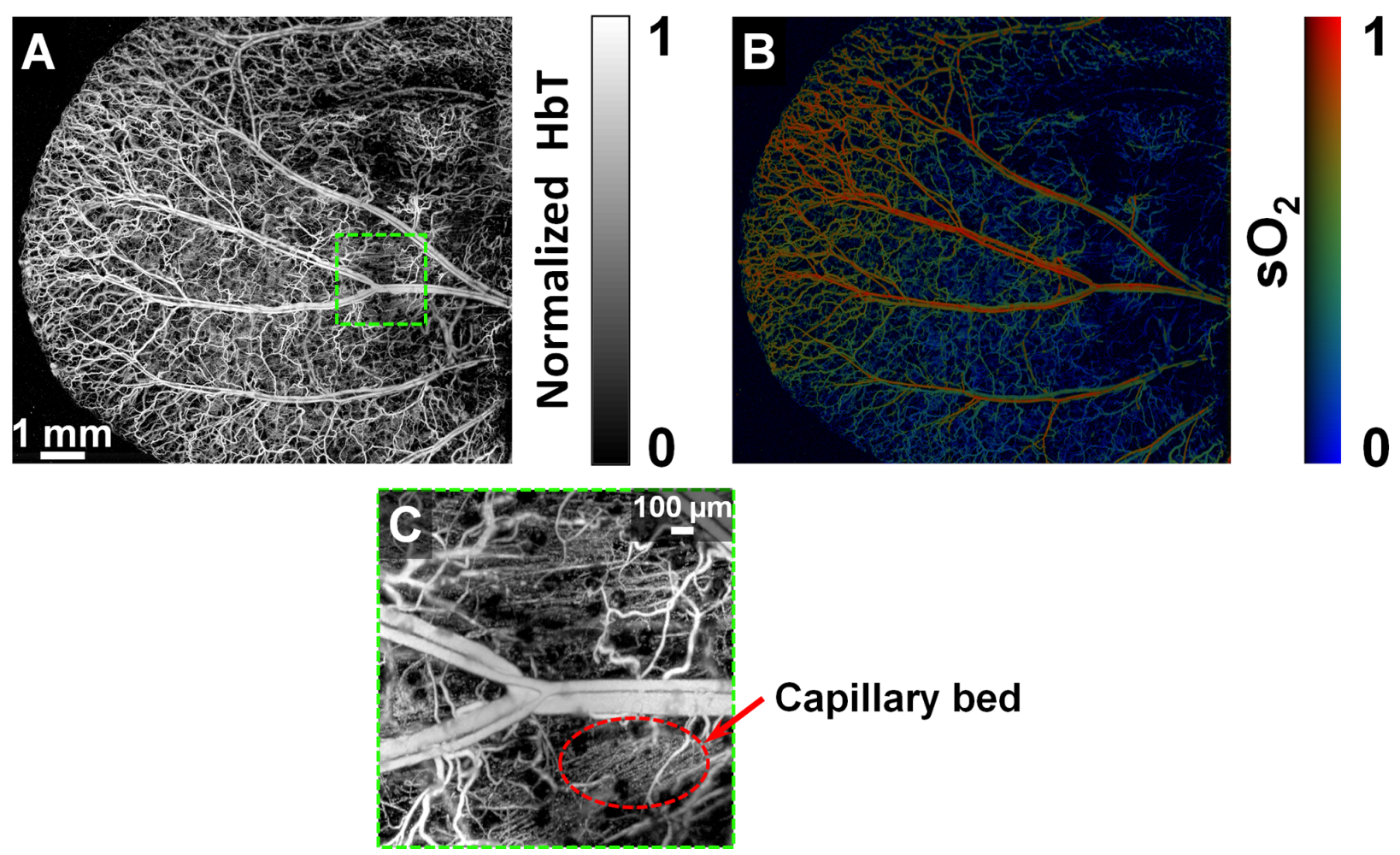

Figure 2. In vivo functional optical-resolution photoacoustic microscopy of a nude mouse ear. MAP images of (A) the total hemoglobin concentration (acquired at $570 \mathrm{~nm}$ ) and (B) the hemoglobin oxygen saturation (acquired at $561 \mathrm{~nm}$ and $570 \mathrm{~nm}$ ). (C) Close-up of the boxed region in panel (A). The scale bar in panel (A) applies to both (A) and (B).

The lateral resolution of the G2 system was experimentally quantified to be $2.6 \mu \mathrm{m}$ by imaging an Air Force resolution test target at $570 \mathrm{~nm}$. Although capable of resolving single capillaries, the high lateral resolution of the G2 OR-PAM also raises a practical challenge: the small source of the photoacoustic wave generates a small amount of acoustic power, which must exceed the noise level to be effectively detected.

Let us consider a small optically absorbing volume, $V$, occupying the focal area of the objective. Due to its small dimension in comparison to the wavelength of the detected acoustic wave, the photoacoustic source can be approximated by a monopole (point) source of ultrasound. The peak power of the generated acoustic wave can be easily calculated [8]. At a distance $r$, the photoacoustic pressure generated by a small optically absorptive object illuminated by a laser pulse of a Gaussian shape $I=\frac{1}{\sqrt{2 \pi}} \frac{F_{0}}{\tau} \exp \left(-t^{2} / 2 \tau^{2}\right)$ can be expressed as [8]:

$$
p=\frac{\sigma \beta F_{0}}{4 \pi \tau^{2} C_{p} r}\left[\frac{-2 t}{\tau \sqrt{2}} \exp \left(-t^{2} / 2 \tau^{2}\right)\right]
$$

where $F_{0}$ is the optical energy fluence, $\beta$ is the isobaric volume expansion coefficient, $\sigma$ is the optical absorption cross section of the absorbing volume, and $C_{\mathrm{p}}$ is the specific heat. For an absorption coefficient $\mu_{\mathrm{a}}$, whose reciprocal is small in comparison to the size of the absorbing volume, $\sigma \approx \mu_{a} V$. Equation (1) can be used to calculate the peak power of the photoacoustic wave on an ultrasonic transducer with a numerical aperture $N A=0.5$ : 


$$
W_{\max } \approx \frac{1}{\pi e \rho c}\left(\frac{\beta \mu_{a} F V}{C_{p} \tau^{2}}\right)^{2} \cdot \frac{N A^{2}}{2} \approx 4 \cdot 10^{-7} \mathrm{~W},
$$

where $\rho$ is mass density and $c$ is the speed of sound. Here we insert in (2) realistic parameters: the optical absorption coefficient of blood $\mu_{a} \sim 300 \mathrm{~cm}^{-1}$, focal volume $V \sim 100 \mu \mathrm{m}^{3}$, which corresponds to an optical resolution of approximately $2.5 \mu \mathrm{m}$, and $F_{0}=20 \mathrm{~mJ} / \mathrm{cm}^{2}$ which is the ANSI safety limit for unintended exposure of the human skin [9]. The full width at half maximum (FWHM) pulse width of the laser pulse was 4.5 ns which gives $\tau=F W H M / 2 \sqrt{2 \ln 2} \cdot \sim 2 n s$.

As one can see, the calculated peak power of the photoacoustic signal is well above the input thermal nose level of ideally matched electrical amplifier:

$$
W_{n}=2 k T \Delta f=4 \cdot 10^{-13} \mathrm{~W},
$$

where $k$ is the Boltzmann constant, $T=300 \mathrm{~K}$ is the ambient temperature, and $\Delta f=10^{8} \mathrm{~Hz}$ is the signal frequency band. That suggests for $2.5 \mu \mathrm{m}$ resolution PAM signal to noise ratio (SNR) about 60dB. However, at near gigahertz frequencies ultrasonic absorption in the tissue and immersion liquid must be taken into account. To reach transducer surface photoacoustic wave propagate distance approximately equal to imaging depth in tissue and much large distance approximately equal to transducer focal length in immersion liquid (water). Ultrasonic attenuation is water is given by $\exp \left(-\alpha f^{2} L\right)$, where $\alpha=25 \cdot 10^{-15} \mathrm{~Hz}^{-2} / m$ is ultrasound absorption coefficient, $f$ ultrasound frequency, and $L=6 \mathrm{~mm}$ is ultrasonic transducer focal length. Taken ultrasonic absorption into account in (1), acoustic pressure at transducer surface is expressed as

$$
p=\frac{\sigma \beta F_{0}}{4 \pi C_{p} L}\left[\frac{-t \sqrt{2}}{\left(\tau^{2}+\alpha L / 2 \pi^{2}\right)^{3 / 2}} \exp \left(-t^{2} / 2\left(\tau^{2}+\alpha L / 2 \pi^{2}\right)\right)\right] .
$$

Corresponding peak power for $\tau^{2}<<\alpha L / 2 \pi^{2}$ reduces to:

$$
W_{\max } \approx \frac{\pi^{3}}{2 e} \frac{1}{\rho c}\left(\frac{\beta \mu_{a} F_{0} V}{C_{p} \alpha L}\right)^{2} \cdot \frac{N A^{2}}{2} \approx 8 \cdot 10^{-8} \mathrm{~W},
$$

which is still much higher than noise level.

Additionally measurable signal peak power must include transducer losses, which for broad-band transducers used in experiment was $31 \mathrm{~dB}$. Correspondingly, a reasonable SNR at ANSI safety limit at light focus can be as high as $22 \mathrm{~dB}$ without signal averaging. ANSI does not regulate fluence level in the focal area of a tightly focused beam, it sets the safety limit for skin exposure for a collimated laser beam at $20 \mathrm{~mJ} / \mathrm{cm}^{2}$. The ANSI limit notwithstanding, previously published works $[10,11]$ have put the safe exposure limit for RBCs to $0.5 \mathrm{~J} / \mathrm{cm}^{2}$ and even higher for other types of cells. That level of fluence should be enough to achieve an near $50 \mathrm{~dB}$ SNR.

Experimentally measured SNR in the acoustic-optical dual foci was $150 \mu \mathrm{m}$ beneath the ear surface measured to be equal to $42.7 \mathrm{~dB}(136.5)$ at laser pulse energy before entering the ear of $80 \mathrm{~nJ}$ and operation wavelength $570 \mathrm{~nm}$. Assume that the thicknesses of epidermis and dermis are $60 \mu \mathrm{m}$ and $90 \mu \mathrm{m}$, respectively. According to [http://omlc.ogi.edu/news/jan98/skinoptics.html], the net epidermal absorption coefficient $\mu_{a_{e p i}}=44 \mathrm{~cm}^{-1}$ and the net dermal absorption coefficient is $\mu_{a_{\text {derm }}}=12.5 \mathrm{~cm}^{-1}$. Thus, the laser fluence at $150 \mu \mathrm{m}$ beneath the ear surface is $0.6 \cdot F_{0}$, where $F_{0}$ is optical fluence on skin surface. Assuming $2.6 \mu \mathrm{m}$ lateral resolution at $150 \mu \mathrm{m}$ beneath the ear surface, the laser fluence in the dual foci is $0.75 \mathrm{~J} / \mathrm{cm}^{2}$. Assuming that blood hemoglobin concentration is $150 \mathrm{~g} /$ liter, and blood has $75 \% \mathrm{HbO}_{2}$ and $25 \% \mathrm{HbR}$ [http://omlc.ogi.edu/spectra/hemoglobin/] the estimated blood absorption coefficient at 570 $\mathrm{nm}$ by is $240 \mathrm{~cm}-1$. Comparing (5) and (3) and including transducer and waveguide losses theoretical estimate gives SNR of $52 \mathrm{~dB}$ which is slightly better than experimentally measured value. Slight discrepancy between thery and experiment can be explained by additional acostic losses in tissue, light reflection from tissue surface and influence of 
limited transducer bandwidth wjich were not taken into account in calculations. Thus, the noise-equivalent sensitivities in absorption coefficient is $\mu_{a_{N E S}}=1.8 \mathrm{~cm}^{-1}$ which is equivalent to molar concentration of the hemoglobin $H b T_{N E S}=16.8 \mu M$ or $4 \cdot 10^{-3}$ of red blood cell volume.

\section{CONCLUSION}

We have developed G2 OR-PAM with high-sensitivity acoustic detection and instrument-scanning. Compared with G1 OR-PAM, the detection sensitivity and the scanning speed are improved by $18 \mathrm{~dB}$ and 5 -fold, respectively. Large-FOV, volumetric microscopy of vascular anatomy and $\mathrm{sO}_{2}$ are demonstrated in vivo.

\section{REFERENCES}

[1] K. Maslov, H. F. Zhang, S. Hu, and L. V. Wang, "Optical-resolution photoacoustic microscopy for in vivo imaging of single capillaries," Opt. Lett. 33(9), 929-931 (2008).

[2] S. Hu, K. Maslov, V. Tsytsarev and L. V. Wang, "Functional transcranial brain imaging by optical-resolution photoacoustic microscopy," J. Biomed. Opt. 14(4), 040503 (2009).

[3] S. Hu, P. Yan, K. Maslov, J.-M. Lee and L. V. Wang, "Intravital imaging of amyloid plaques in a transgenic mouse model using optical-resolution photoacoustic microscopy," Opt. Lett. 34(24), 3899-3901 (2009).

[4] S. Hu, B. Rao, K. Maslov and L. V. Wang, "Label-free photoacoustic ophthalmic angiography," Opt. Lett. 35(1), 13 (2010).

[5] S. Oladipupo, S. Hu, A. Santeford, J. Yao, J. R. Kovalski, R. Shohet, K. Maslov, L. V. Wang, and J. M. Arbeit, "Conditional HIF-1 induction produces multistage neovascularization with stage-specific sensitivity to VEGFR inhibitors and myeloid cell independence", Blood (in press).

[6] S. Hu, K. Maslov and L. V. Wang, "In vivo functional chronic imaging of a small animal model using opticalresolution photoacoustic microscopy," Med. Phys. 36(6), 2320-2323 (2009).

[7] S. Hu, K. Maslov and L. V. Wang, "Second-generation optical-resolution photoacoustic microscopy with improved sensitivity and speed," Opt. Lett. (Under review).

[8] I. G. Calasso, W. Craig, and G. J. Diebold, "Photoacoustic point source" Phys. Rev. Lett. 86(16), 3550-3553 (2001).

[9] “American national standard for the safe use of lasers Z136.1," American National Standards Institute, New York, (2000).

[10] V. P. Zharov, E. I. Galanzha, E. V. Shashkov, N. G. Khlebtsov, and V. V. Tuchin, "In vivo photoacoustic flow cytometry for monitoring of circulating single cancer cells and contrast agents," Opt. Lett. 31(24), 3623-3625 (2006).

[11]D. O. Lapotko, and V. P. Zharov, "Spectral evaluation of laser-induced cell damage with photothermal microscopy," Lasers Surg. Med. 36(1), 22-30 (2005). 